\title{
Dose-response curve of a microfluidic magnetic bead-based surface coverage sandwich assay
}

\author{
Matteo Cornaglia, Raphaël Trouillon, H. Cumhur Tekin ${ }^{+}$, Thomas Lehnert, and Martin A. M. \\ Gijs*
}

Laboratory of Microsystems, Ecole Polytechnique Fédérale de Lausanne, CH-1015 Lausanne, Switzerland

+ present address: Canary Center at Stanford for Cancer Early Detection, Department of Radiology/ School of Medicine, Stanford University, Stanford, USA

*E-mail: martin.gijs@epfl.ch

\begin{abstract}
Magnetic micro- and nanoparticles ('magnetic beads') have been advantageously used in many microfluidic devices for sensitive antigen $(\mathrm{Ag})$ detection. Today, assays that use as read-out of the signal the number count of immobilized beads on a surface for quantification of a sample's analyte concentration have been among the very sensitive ones and have allowed protein detection lower than the fg $\mathrm{mL}^{-1}$ concentration range. Recently, we have proposed in this category a magnetic bead surface coverage assay, ${ }^{1}$ in which 'large' $(2.8 \mu \mathrm{m})$ antibody $(\mathrm{Ab})$-functionalized magnetic beads captured their $\mathrm{Ag}$ from a serum and these Ag-carrying beads were subsequently exposed to a surface pattern of fixed 'small' $(1.0 \mu \mathrm{m}) \mathrm{Ab}$-coated magnetic beads. When the system was exposed to a magnetic induction field, the magnet dipole attractive interactions between the two bead types were used as a handle to approach both bead surfaces and assist with the Ag-Ab immunocomplex formation, while unspecific binding (in absence of an $\mathrm{Ag}$ ) of a large bead was reduced by exploiting the viscous drag flow. The dose-response curve of this type of assay had two remarkable features: (i) its ability to detect an output signal (i.e. the bead number count) for very low $\mathrm{Ag}$ concentrations, and (ii) an output signal of the assay that was nonlinear with respect to the Ag concentration. We explain here the observed dose-response curves and show that the type of interactions and the concept of our assay is in favor of detecting the lowest analyte concentrations (where typically either zero or one Ag is carried per large bead), while larger concentrations are less efficiently detected. We propose a random walk process of the Ag-carrying bead over the magnetic landscape of small beads and this model description allows explaining the enhanced overall capture probability of this assay and its particular non-linear dose response curves.
\end{abstract}




\section{Introduction}

Measuring Ags at very low concentration in blood or serum samples allows early disease diagnosis and is important for proposing more accurate medical treatments that allow increasing the survival rates of the ${ }_{5}$ patients. ${ }^{2}$ Detection of a disease by measuring a specific Ag in a serum matrix is often the preferred minimallyinvasive solution. Very sensitive nucleic acid detection is enabled by polymerase chain reaction techniques, but there is no comparable method that can be used to 'amplify' proteins, ${ }^{3}$ which necessitates the development of very sensitive types of assays. Moreover, blood contains, besides the protein that is the Ag of interest, many different molecules varying over a huge amount of concentrations (in the $\mathrm{pg} / \mathrm{mL}-\mathrm{mg} / \mathrm{mL}$ range). ${ }^{4,5}$ While, 10 traditionally, protein purification is obtained starting from milliliter blood samples to increase the number of molecules to be detected, microsystems techniques enable usage of much lower blood sample consumption. However, detecting low-concentration biomarkers in small sample volumes evidently may be an issue and would require developments of analytical techniques with ultrahigh sensitivity. A lot of the microfluidics literature of recent years has focused therefore on improving the limit of detection (LOD), i.e. decreasing the is lowest concentration at which the presence of the Ag can be detected, and a truly impressive progress has been noted. ${ }^{6-8}$ Often, these techniques proved to be not accurate for the lowest concentrations and follow-up work will focus now on the most promising assays, so that also the limit of quantification (LOQ), ${ }^{9}$ i.e. the limiting concentration at which the Ag can still be accurately measured, will be decreasing in future.

Among the most sensitive surface coverage immunoassays, which use evaluation of the number of immobilized ${ }_{20}$ beads on a surface for quantification of a sample's analyte concentration, S. V. Mulvaney et al. ${ }^{10}$ presented magnetic bead-based assays that were denominated as sequential and semi-homogenous assays. The target Ags in a sequential assay, and subsequently the detection Abs, were incubated on the wall of a microfluidic chamber that carried capture Abs. Hereafter, magnetic beads functionalized with detection Abs were transported over the detection surface, and so-called force discrimination was in a buffer flow was exploited to detach unspecifically${ }_{25}$ bound beads. $100 \mathrm{fg} \mathrm{mL}^{-1}$ of staphylococcal enterotoxin B (SEB) could be detected in $2 \mu \mathrm{L}$ phosphate buffer saline (PBS). Alternatively, for the semi-homogenous assay configuration, the beads were pre-mixed with target Ags and subsequently they were transported over the detection area. The semi-homogenous assay had a LOD of $1 \mathrm{fg} \mathrm{mL}{ }^{-1}$ SEB. Morozov et al. ${ }^{11}$ described instead sequential force flow discrimination assays. Here, the target molecules were concentrated on a detection area by applying an electrophoretic force. Then, magnetic beads ${ }_{30}$ conjugated to detection Abs were led over the Ag-carrying detection area in presence of a magnetic field. The assay showed a LOD as low as $1 \mathrm{fg} \mathrm{mL}^{-1}$ for streptavidin spiked in buffer. S. J. Osterfeld et al. ${ }^{12}$ also proposed a sequential assay principle. In this work, first target Ags were immobilized on a surface and were linked to biotinylated detection Abs; subsequently streptavidin-coated magnetic beads were transported over the thus prepared surfaces. During this procedure, magnetic beads that were attached on the detection surface via ${ }_{35}$ streptavidin-biotin bonds could be live-monitored via giant magnetoresistance-based sensors, whose electrical resistances change in the presence of beads. ${ }^{13}$ The study resulted in a $200 \mathrm{fg} \mathrm{mL}^{-1}$ LOD for carcinoembryonic 
antigen. ${ }^{14}$ Moreover, signal amplification permitted to lower the LOD to $10 \mathrm{fg} \mathrm{mL} \mathrm{m}^{-1}$. Furthermore, for most of these assays, the dose-response curve is largely expected to be described by a Langmuir relationship. Indeed, the sample is first injected over a substrate decorated with $\mathrm{Ab}$ which will bind the Ag. The amount of bound $\mathrm{Ag}$ is here expected to be described by a Langmuir relationship. In a second step, a large quantity of magnetic beads is ${ }_{s}$ injected in the device and is expected to bind to all of the immobilized Ag. As a consequence, in the case where the $\mathrm{Ag}$ is first bound of the walls of the microchannel, the calibration curve is expected to be described by the Langmuir relationship.

Previously, we have demonstrated a microfluidic method for ultra-sensitive protein detection, in which first 'large' $(2.8 \mu \mathrm{m})$ Ab-functionalized magnetic beads specifically captured Ags from a serum matrix under active ${ }_{10}$ microfluidic mixing. ${ }^{1}$ Subsequently, the large beads loaded with the Ags were exposed to a surface pattern of fixed 'small' $(1.0 \mu \mathrm{m})$ Ab-coated magnetic beads. During this exposure, attractive magnetic bead dipole-dipole interactions improved the contact between the two bead types and helped the Ag-Ab immunocomplex formation. Finally, the Ag concentration was detected by counting the number of surface pattern-bound large magnetic beads. A LOD of only 200 molecules of Tumor Necrosis Factor- $\alpha$ (TNF- $\alpha$ ) in a serum sample volume 15 of $5 \mu \mathrm{L}$, corresponding to a concentration of $1 \mathrm{fg} \mathrm{mL}^{-1}$, was demonstrated. In the present manuscript, we present a theoretical model that actually explains both the very low detection limit obtained in this kind of assay and the particular shape of its dose-response curve.

\section{Materials and Methods}

\section{Microfluidic chip and assay protocol}

Briefly, the reported assay was performed in a polydimethylsiloxane (PDMS) microfluidic chip featuring microfluidic channels, pneumatic valves and an integrated mixer. ${ }^{15}$ This device was connected through ports to external syringe pumps (Nemesys - Cetoni, Korbussen, Germany) for automated fluidic manipulations All the different microfluidic device operations (e.g.: valve opening/closing, on-chip mixing) were handled via a home${ }_{25}$ built Labview (National Instruments, Austin TX, USA) program. Optical micrographs were taken with a Pixelink PL-P742 digital camera that was linked via a $0.5 \times$ TV adapter (Zeiss product no. 456101) to an Zeiss Axiovert S100 inverted microscope, which was provided with a $32 \times$ Zeiss LD-Achroplan magnification objective.

The detection area of our chip was represented by a $250 \mu \mathrm{m}$ x $2500 \mu \mathrm{m}$ pattern of Ab-functionalized $1 \mu \mathrm{m}$ ${ }_{30}$ superparamagnetic beads, which were immobilized on a glass substrate via electrostatic self-assembly on aminopropyl-trietoxysilane (APTES) dot structures. ${ }^{16}$ Larger Ab-coated magnetic beads (diameter $2.8 \mu \mathrm{m}$ ) were used for specifically capturing target Ags from the sample solution under analysis. More specifically, for each assay, $\sim 3500$ 'large' large beads were introduced to the mixing chamber together with a $5 \mu \mathrm{L}$ sample solution and active on-chip mixing was performed to extract the target Ags from the sample via specific binding to the ${ }_{35}$ large beads' surface. Ag-carrying beads in buffer were then transported towards the detection zone, through a 
$250 \mu \mathrm{m}$ wide, $60 \mu \mathrm{m}$ high microfluidic channel. The sandwich immunoassay was performed by magnetophoretically exposing the small bead pattern to the large beads by placing the chip in a $27 \mathrm{mT}$ magnetic field, as generated by an external permanent magnet. The small magnetic bead pattern on the chip leaded to local magnetic field maxima, enhancing interaction between the immobilized small beads and the moving large ${ }_{5}$ ones, through magnetic dipole-dipole forces (Fig. 1a). The improved selectivity of the small bead-large bead binding was based on the fine-tuning of the balance between magnetic dipolar interactions and drag forces, which allowed selectively removing non-specifically bound beads, i.e. beads that were not linked via Ag ${ }^{17}$ (Fig. $1 b)$.

Subsequently, simply counting the large beads immobilized on the pattern of small beads provided the detection ${ }_{10}$ signal that allowed quantification of the Ag concentration (Fig. 1c). As an example of the obtained results, the reported dose-response curve for biotinylated anti-streptavidin that was used as 'Ag' spiked in $5 \mu \mathrm{L}$ serum is shown in Fig. 1d, as already reported in our previous work. ${ }^{1}$. Protein detection experiments were performed by preparing and testing fetal bovine serum (FBS) samples containing progressively lower concentrations of target Ags. The number of Ag molecules corresponding to each Ag concentration is simply calculated by considering is the molecular weight of the molecule of interest.

\section{Chemicals and materials}

$\mathrm{Si}$ and float glass wafers (4-inch $550 \mu \mathrm{m}$ thick), de-ionized water were provided by the Center of Micro- and Nanotechnology of EPFL. Negative photoresist GM 1075 SU-8 was obtained from Gersteltec (Pully, Switzerland). AZ ECI 3027 positive photoresist was from AZ Electronic Materials (Wiesbaden, Germany). ${ }_{20}$ PDMS Sylgard 184 was purchased from Dow Corning (Wiesbaden, Germany). The microfluidic chip was composed of four bonded PDMS layers, each replicated using a different SU-8 master microstructure on a $\mathrm{Si}$ wafer. SU-8 was patterned using standard lithography. The solution of APTES (product no. 440140), 10× concentrate solution of phosphate buffered saline (PBS) (product no. 5493), Tween-20 (product no. 1379) and biotin (product no. B4501) were acquired from Sigma-Aldrich (Buchs, Switzerland). Biotinylated goat anti${ }_{25}$ Streptavidin and streptavidin were purchased from Vector Laboratories (Reactolab SA, Servion, Switzerland). Dynabeads MyOne Streptavidin C1 (small beads), Dynabeads M-280 Streptavidin (large beads), and fetal bovine serum (FBS) dialyzed were obtained from Life Technologies (Zug, Switzerland). FBS standard quality was purchased from PAA Laboratories (Chemie Brunschwig AG, Basel, Switzerland) and Pluronic F-127 was purchased from BASF (Basel, Switzerland). A PBS (0.15 M NaCl, 0.01 M phosphate, $\mathrm{pH}$ 7.4) solution was ${ }_{30}$ acquired by diluting a $10 \times$ concentrated PBS solution. PBS-Tween solution (PBST $\mathrm{n} \%$, with $\mathrm{n}$ a number) was made by diluting $\mathrm{n} \%$ (volume/volume) Tween-20 within PBS. A buffer solution was prepared by dilution of $0.08 \%$ (weight/volume) Pluronic F127 in the PBS. To reach the experimental ligand concentration levels, biotinylated anti-streptavidin dilutions in PBS or FBS dialyzed were prepared. 


\section{Results and Discussion}

Enhancement of the large bead capture efficiency

During their passage through the detection area, large beads "scan" the immobilized beads pattern by repeatedly s interacting with their Ab-functionalized surface. The distance between two immobilized beads has been estimated as $\sim 12 \mu \mathrm{m}$, and the large beads slide at a constant speed $\left(\sim 1 \mathrm{~mm} \mathrm{~s}^{-1}\right)$ over the surface of the device, so that the time between two large bead/ immobilized bead interactions is estimated as $12 \mathrm{~ms} .{ }^{18}$ Each large bead can be considered, in first approximation, as a sphere, of which only a fraction of the surface is covered by Ags and can be specifically bound to the Abs on the small beads. Ideally, capture is immediately triggered when an ${ }_{10}$ Ag-covered part of the surface encounters the small bead pattern. The probability to successfully bind the large bead, $P_{\text {capture, }}$ is therefore directly related to the capability of the device to intercept this small area over the whole bead surface. Two different cases can now be considered.

In the absence of immobilized beads, each large bead would merely slide over the glass surface. The vertical orientation of the bead is in fact pinned by the presence of the $27 \mathrm{mT}$ vertical magnetic field at the glass surface, is and, assuming a perfectly spherical geometry, the contact between the large bead and the sensing substrate would be reduced to a single point, over the duration of the whole experiment. ${ }^{19}$ The binding probability can therefore be reduced to the probability that this contact point exactly corresponds to the location of the adsorbed Ags. $P_{\text {capture }}$ is then proportional to the ratio of the part of the bead surface that is effectively covered with Ags over the whole bead surface ${ }^{20}$ :

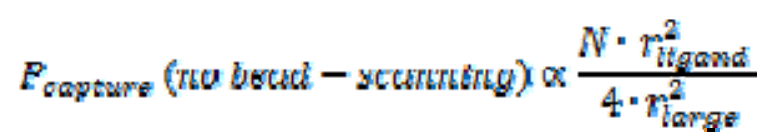

${ }_{20}$ with $N$ the number of Ags on the large bead surface, $r_{\text {ligand }}$ the average radius of a ligand molecule (for the biotinylated anti-streptavidin - streptavidin couple, it is more appropriate to speak of a ligand-receptor rather than an Ag-Ab system) and $r_{\text {large }}$ the radius of the large bead. This probability is evidently extremely low for single molecule carrying beads. We refer to this case as the 'no bead-scanning' case.

${ }_{25}$ In our assay, the presence of the small bead pattern introduces instead what we define as "bead-scanning" mechanism. In our device, the external magnetic field still vertically pins the magnetic moment of the large beads, but their lateral orientation is induced to vary continuously, at each encounter with any of the $N_{\text {dots }}$ pattern dots. Hence, a progressively increasing portion of the large bead surface gets in contact with the functionalized surface of the small beads (Fig. 2a). ${ }^{20}$ If $P_{\text {capture }, i}$ is the probability that the large bead is captured on the $\mathrm{i}^{\text {th }}$ dot of ${ }_{30}$ small beads, we can write that:

$$
P_{\text {oapture }}(\text { bead }-\operatorname{scanning}) \propto \sum_{i=1}^{N_{\text {diacs }}} P_{\text {oapturst }}
$$


Given the sequential scanning of the large bead by the array of dots, a binding at the $\mathrm{i}^{\text {th }}$ dot is equivalent to (i-1) non-binding encounters with the (i-1) previous dots in the trajectory of the large bead, followed by a successful encounter on the $\mathrm{i}^{\text {th }}$ dot. If we introduce $P_{S B}$, the probability of a successful binding of the large bead upon an encounter with a small bead dot, we obtain that:

$$
P_{\text {oapturet }} \propto P_{S Q}\left(1-P_{S Q}\right)^{t-1}
$$

${ }_{5}$ Hence, $P_{\text {capture }}$ can be written as:

$$
P_{\text {oxpture }}(\text { bead scanning }) \times P_{S E} \sum_{i=0}^{N_{d i p t s}-1}\left(1-P_{S E}\right)^{t}=1-\left(1-P_{S E}\right)^{N_{d a t s}}
$$

$P_{S B}$ is the factor indicating the probability of $\mathrm{Ag}-\mathrm{Ab}$ binding upon an inter-bead encounter. In the considered case, it corresponds to the ratio between the fraction of surface scanned during a large bead/ small bead interaction and the total surface of the large bead. Therefore,

$$
P_{S E}=\frac{N \cdot 8 \cdot r_{\text {itgamd }}}{\pi \cdot r_{\text {targe }}}
$$

${ }_{10}$ where $\delta$ is the angle intercepting the arc formed by the point of contact during the large bead/ small bead interaction, with:

$$
\delta=\operatorname{arcos}\left(\frac{\eta_{\text {ange }}-r_{\text {sman }}}{n_{\text {arge }}+r_{\text {sman }}}\right)
$$

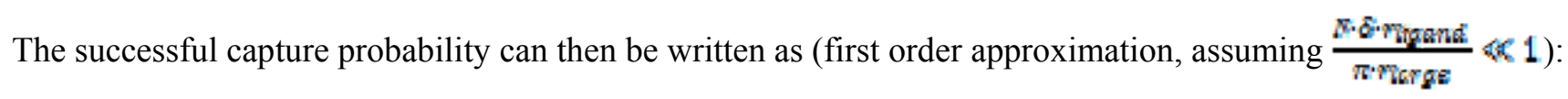

$P_{\text {oxpture }} \propto \frac{N \cdot \bar{\delta} \cdot r_{\text {thand }}}{\pi \cdot r_{\text {lorge }}} \cdot N_{\text {dotg }}$

In our system, we experimentally observed that each large bead interacts, in average, with about 200 pattern dots during its passage through the whole detection area, i.e. $\mathrm{N}_{\text {dots }} \sim 200$.

${ }_{15}$ In Fig. 2b, $P_{\text {capture }}$ has been calculated using equation (1) ('no bead-scanning') and equation (4) ('beadscanning'), taking $N=1$ and $\eta_{\text {tgam }}=2.2 \mathrm{~nm}$, showing indeed the dramatically higher $P_{\text {capture }}$ obtained from the bead-scanning system. In Fig. 2bi, the radius of the small bead is fixed at $0.5 \mu \mathrm{m}$, and the large bead radius is varied. This analysis shows that increasing the radius of the large bead decreases the probability of capture, because it increases the large bead's surface, therefore reducing the probability of a successful encounter ${ }_{20}$ between the ligand and the receptor. In Fig. 2bii, this time the radius of the large bead is fixed at $2.8 \mu \mathrm{m}$, and the radius of the small bead is varied. The 'no bead-scanning' case shows no variation, as this model does not take the small beads into account. As expected, if the bead-scanning is enabled, increasing the size of the small bead 
improves the capture efficiency, as a larger section of the large bead is explored at each interaction with a small bead, from the definition of $\delta$. It is worthy to notice that, in these first estimations, the capture probability enhancement has been calculated by assuming only lateral rotation of the large beads, at each interaction with a small bead dot. However, we cannot exclude that further large bead reorientations occur between successive ${ }_{5}$ collisions with pattern dots. Moreover, even vertical flipping and rolling of the large beads are very plausible events in our system, likely triggered by temporary unspecific binding events and driven by the strong shear forces acting on the large beads under the presence of fast flow in the microchannel.

This first model is aimed at emphasizing the importance of the scanning mechanism in improving the detection. This is a simple model, principally taking into account geometrical considerations. However, our main purpose 10 is to use this system as an analytical system, to detect low levels of Ag. A second analytical model aiming at understanding and predicting the calibration curves is therefore needed. In the next sections, rather than the mere scanning mechanism, the topic of interest is the relation between the output signal and the concentration of $\mathrm{Ag}$ injected in the device.

\section{is Analytical model for the dose-response curve}

Intuitively, it is already clear that this type of assay will be more efficiently detecting low Ag concentrations than higher concentrations. Indeed, if a few thousand large beads each carry one Ag, they theoretically could all be specifically bound to the small bead pattern and the large beads could be counted as representing the number of Ags. On the other hand, suppose now that the large beads had been mixed in the mixing chamber with a ${ }_{20}$ higher concentration sample and that each large bead would carry 10 or $100 \mathrm{Ags}$; in this case, at best, all large beads could be linked to the small bead pattern, so that these higher concentrations would be less efficiently detected indeed. The logic consequence of this is that the output signal of our assay (i.e. the count of the number of large beads) will not be a linear function of the number of Ags present in the sample. However, in order to obtain an analytical expression for the dose-response curve, a more rigorous treatment is necessary.

${ }_{25}$ For the reader's convenience, we present here the main reasoning behind the model, as mentioned and based on previously reported results. ${ }^{20-22}$ We assume first that the binding probability of the large bead $P_{b}(l)$, as a function of the distance $l$ travelled in the microchannel along the small bead landscape, satisfies a $1^{\text {st }}$ order reaction kinetics law

$$
P_{2}(b)=P_{0}\left(1-e^{-i / 2}\right)
$$

where $L$ is the characteristic distance of successful binding and $P_{0}$ the probability of binding for a large bead for ${ }_{30} t \rightarrow+\infty$. In principle, the factor $P_{Q} \leq 1$ can be evaluated from the geometrical aspects of the system. In our system $l<<L, P_{b}(l)$ can be linearized to $P_{0} l / L{ }^{20}$ This arises from the low fraction $(<2 \%)$ of beads captured over the length of the channel, thus confirming that the binding probability is low and that $l<L$. As expected, a 
linear-like behaviour is observed for the large bead count over the length of the detection zone. ${ }^{20}$ As a consequence, the variations of the captured fraction vs. $l$ can be directly related to $P_{b}(l)$ and hence to the inverse of the characteristic length $L$. Furhermore, it has been previously shown that the bead slides over the substrate at an average speed $v_{0}$ in between the small bead dots. This observation allows to relate the value of $L$ to a ${ }_{5}$ characteristic binding time $\tau \equiv L / \tau_{0}$.

A random walk model of the contact point on the large bead's surface is used to describe the interactions between the large bead and the immobilized small bead dots (Figure 3a). This contact point is stochastically displaced over the large bead surface until it finds an $\mathrm{Ag}$ that is bound to the large bead. The duration of this random walk, until the large bead is captured, is expected to be related to the mean first passage time $\left(t_{M F P}\right)$ for a ${ }_{10}$ point randomly walking over the surface of a sphere from a starting point to a target position, which can be generally expressed as ${ }^{23}$

$$
t_{M F P} \propto A+B \ln (d)
$$

where $d$ is a dimensionless distance separating the starting point of the walk on the sphere from its target position, and $A$ and $B$ are constants, and where it can be proven that the factor $A$ is significant only for low $d^{23}$. For low densities of bound Ags on the large bead surface, the random walk length $d$ necessary to encounter an ${ }_{15} \mathrm{Ag}$ is big, as assumed above $(k<L)$, and we therefore assume that the contribution of $A$ can be neglected. To derive the distance $d$, we consider an uniform distribution of $N_{A g, b o u n d}$ points, which correspond to locations of Ags bound on the surface of a sphere. We assume here a homogeneous distribution of Ag over the surface of the bead with radius $r_{\text {large, }}$ leading to ${ }^{24,25}$

$\operatorname{dn}\left(\frac{8 \pi}{\sqrt{3}}\right)^{1 / 2} \frac{\eta_{\text {arge }}}{\sqrt{N_{\text {Aq.boumd }}}}$

so that

$$
5_{M F P} \times \ln \left(Y N_{A Q, 5 o u m d}\right)
$$

${ }_{20}$ where $\gamma$ is a constant. To express $N_{A g, b o u n d}$ as a function of $N_{A g}$, i.e. the total number of Ags in the sample, we assume a Langmuir relationship between these two values, ${ }^{26,27}$ with $\alpha$ being a constant (Fig. 3b):

$$
N_{A Q, \text { bound }}=\frac{\propto x N_{A \sigma}}{1+e x N_{A G}}
$$

The Langmuir relationship typically describes the amount of molecules adsorbed onto a surface in equilibrium with a solution of known concentration. Above a certain concentration of Ag, the response saturates, as all the possible adsorption sites are occupied. For low amounts of Ag molecules, the relation between amount of free 
and bound $\mathrm{Ag}$ is linear. The parameter $\alpha$ controls the $\mathrm{Ag}$ concentration at which the system switches from the linear to the saturated response. It largely accounts for the affinity of the ligand to the receptor as well as the mixing efficiency in the chip. The system saturates at lower Ag concentrations for larger $\alpha$ values. Fig. $3 \mathrm{~b}$ shows profiles obtained from equation (12) for different values for $\alpha$.

${ }_{5}$ Finally, $t_{M F}$ can be expressed as a function of the experiment-derived time $\tau$, i.e. the characteristic time at which a large bead carrying an Ag would have been effectively linked to the small bead pattern. The probability that the bead is captured exactly at the time $t$, the instantaneous capture probability, $p_{c}(t)$ is introduced. This probability is nothing else but the probability density function of $P_{b}(t)$ :

$p_{v}(t)=\frac{d P_{b}}{d t}(t)$

It is assumed that the first encounter between, on the one hand, the point of contact between a large and a small 10 bead and, on the other hand, an Ag bound to the large bead. Therefore, the first passage of this point of contact at the position of an Ag results in the binding of the large bead. Hence, $t_{M F P}$ can be evaluated from $p_{c}(t)$ following

$t_{M F P}=\int_{0}^{+\infty} u p_{0}(u) d u=\int_{0}^{+\infty} u \frac{d P_{b}}{d u}(u) d u$

By solving the integral, and using the expression for $P_{b}(t)$, we find that $t_{M F P}=P_{0} \tau$ and $t_{M F P}$ is hence directly proportional to $\tau$, so that:

$$
\frac{P_{Q}}{L}=\frac{P_{Q}}{\tau v_{0}}=\frac{P_{0}^{2}}{t_{M F P} v_{Q}}
$$

is and the total number of bound large beads as a function of $N_{A g}$, i.e. output $\left(N_{A g}\right)$, can be written as:

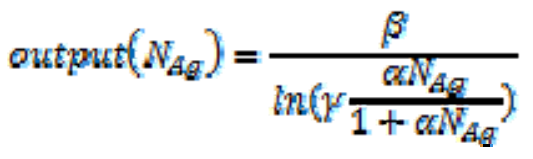

where $\beta$ is a negative constant. For $N_{A g}=0$, an output value of 0 is obtained.

Fig. 4a,b,c shows plots of equation (16) for different conditions. The experimental data was fit to the model defined in equation (16), as shown in Fig. 4d, returning the following values for the fitting parameters: $\alpha=$ $1.3 \times 10^{-12}$ molecule ${ }^{-1}, \beta=-0.15, \gamma=2.6$. These parameters were used to compute with one of the parameters being ${ }_{20}$ varied ( $\alpha, \beta$ and $\gamma$ on panels $\mathrm{a}, \mathrm{b}$ and $\mathrm{c}$ in Fig. 4, respectively), the other ones being fixed to the values obtained from the experimental fit. Even though it is possible to explain the contribution of different physical phenomena 
to the parameters $\alpha, \beta$ and $\gamma$, it is not possible to provide an exact expression for these values. Indeed, most of the equations shown above describe relations of proportionality rather than equality, and some parameters, such as $\alpha$ or $L$, are not fully described by an exact equation. As a consequence, fitting the experimental results to the described model is the only way to obtain estimations.

s This analysis shows that $\alpha$, which is obtained from the Langmuir equation, controls the concentration from which the Ag coverage over the bead is sufficiently high, so that the assumption $l<L$ is not satisfied anymore. As shown in Fig. 4a, the curves actually diverges towards $+\infty$ for $N_{A q}=1 /(\gamma-1) \%$. The parameter therefore accounts for the binding affinity and the mixing efficacy in the system. The effect of the negative parameter $\beta$ is shown in Fig. 4b. Here, no obvious effect can be observed, as this is merely a multiplication constant which ${ }_{10}$ scales the magnitude of the response. More importantly, it is expected that this value depends on $P_{0}$ and $v_{0}$, from equation (15), and more generally on the beads size. Indeed, through the intercept angle $\delta$, these can modulate the surface of the large bead that is accessible to the small bead, and therefore the overall capture probability. This parameter also accounts for the random walk over the bead surface (equation 9). In this case, a slower random walk (or a larger $t_{M F P}$ ) would indicate a larger parameter $B$ in equation 9 , and a smaller $\beta$. This would ${ }_{15}$ result in a lower output, at fixed $N_{A g}$. This also corresponds, qualitatively, to an increase in $L$.

Finally, in the case of $\gamma$ (Fig. 4c), a behavior similar to $\alpha$ is observed. High $\gamma$ values induce a divergence of the curve for lower $N_{a g}$. From equation 11, $\gamma$ accounts for the distribution of the ligands over the large bead surface (equation 10). It is therefore determined by the geometry of the large bead. Large $\gamma$ values therefore indicate a faster saturation of the large bead surface with $\mathrm{Ag}$ because of a shorter inter-adsorbed $\mathrm{Ag}$ distance $d$ (equations 2010 and 11).

As stated above, this model is valid only for low Ag amounts. At higher concentrations, equation 8 cannot be linearized anymore, and a different expression for the output $\left(N_{A g}\right)$ function is expected. Moreover, the $\mathrm{Ag}$ concentration is largely assumed to be a continuous function in the model, whereas it actually describes a low number of discrete molecules. The result from this fact is that the response of the system is discrete too. For ${ }_{25}$ instance, if only one Ag is injected, only two results can theoretically be obtained for output $\left(N_{A g}\right), 0$ and 1/3500 (as 3500 large beads were injected). In this case, it is expected that the result obtained from output $\left(N_{A g}\right)$ would then be better described by the average of several experiments. We can therefore speculate that, at very low Ag amounts, the output of the assay would be seen as a stochastic variable with two states (output $\left(N_{A g}\right)=0$ and $\left.\operatorname{output}\left(N_{A g}\right)=1 / 3500\right)$ and the model would return the expected value of this random variable. Technically, this ${ }_{30}$ case was not encountered in our analysis, as the LOD of the assay was found to be $\sim 200 \mathrm{Ag}$ and the signal obtained for this value is at least an order of magnitude above $1 / 3500$. 


\section{Conclusions}

A theoretical description of the dose-response curve of a microfluidic magnetic bead-based surface coverage sandwich assay was presented. This model was found to fit well the experimental data, explaining the sub-linear ${ }_{s}$ behaviour of the system. Furthermore, this analysis emphasizes the basic mechanisms controlling this type of assays. As the ligand and receptors are bound to surfaces, it is critical to increase the probability that they encounter each other, thus triggering recognition and a capture event. Simulating the trajectory of the point of contact, with a random-walk (or any other well-chosen model), can account well for the behaviour of the system. This fact also suggests that the mechanism of the whole chip can, under certain assumptions, be reduced 10 to this stochastic motion of the contact point. Our modeling approach allows moreover clearly identifying the experimental factors which determine the sub-linear behaviour of the system, thus readily suggesting how to modify the assay to further to enhance its performance. In these terms, three main experimental modifications are foreseen to improve the system: (i) the length of the detection area will be increased, for a more efficient capture of the Ag-carrying large beads; (ii) longer on-chip mixing times will be used to enhance the efficacy of ${ }_{15} \mathrm{Ag}$ extraction from the sample and specific binding on the large beads; (iii) the size of large beads will be reduced (and/or the one of small beads will be enlarged), to ensure a larger contact surface for the scanning mechanism and, therefore, higher probability of specific bead binding.

Several so-called magnetic surface coverage immunoassays based on fluidic flow discrimination have now shown extreme sensitivity. ${ }^{1,10,11}$ We think that the possibility of fine-tuning immunocomplex-induced binding ${ }_{20}$ between magnetic beads and the surface is key to these findings. Also these systems exploit the viscous drag forces, as imposed by the flow, to wash non-specifically attached beads from the detection area. Equally well important are the rapid dynamic extraction of target Ags from the original raw sample, as is the subsequent processing of the magnetic beads in a clean buffer solution, which are factors that are in favour of reproducibility and accuracy of the assay results. A possible disadvantage of many ultrasensitive protein ${ }_{25}$ detection techniques is the need to use more complex and sophisticated assay protocols; however, we think that the mentioned assays that provide the assay detection signal via a magnetic bead surface coverage will provide interesting options for future accurate and sensitive immunoassays. Besides sensitivity, robustness, large dynamic range, low-cost, accuracy and the possibility of multiplexing represent important criteria that will be key in deciding on the success of future analytical systems, and these mostly outstanding issues will have to be ${ }_{30}$ addressed in future.

\section{Acknowledgements}

We thank the European Commission for financial support in the Biomax project (ITN 264737) and in the EU Ideas program (ERC-2012-AdG-320404). This research was supported by the Ecole Polytechnique Fédérale de Lausanne and we would like to thank the staff of the Center of Micro- and Nanotechnology of EPFL for ${ }_{35}$ assistance in the microfabrication. 


\section{Figures}

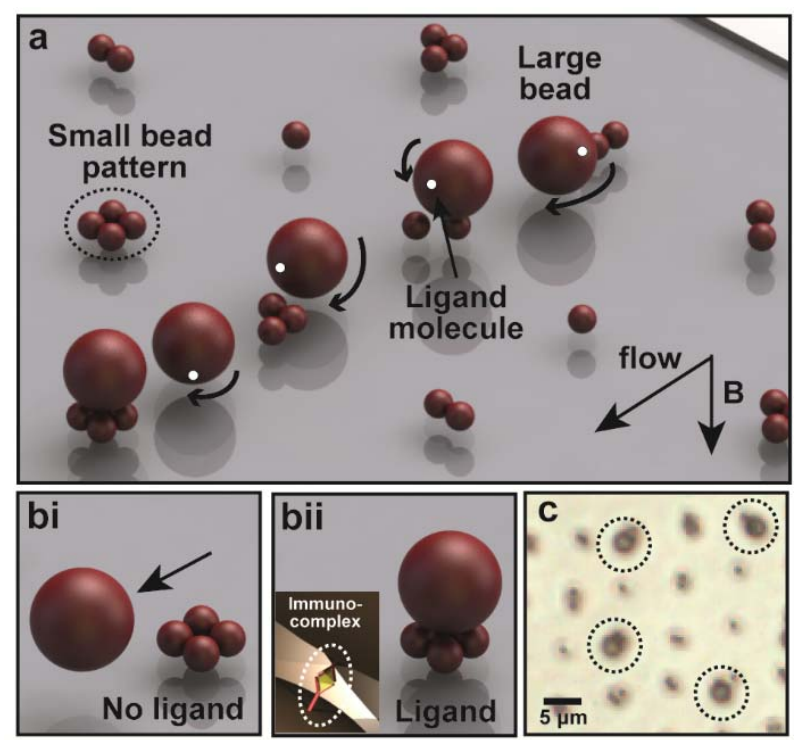

d

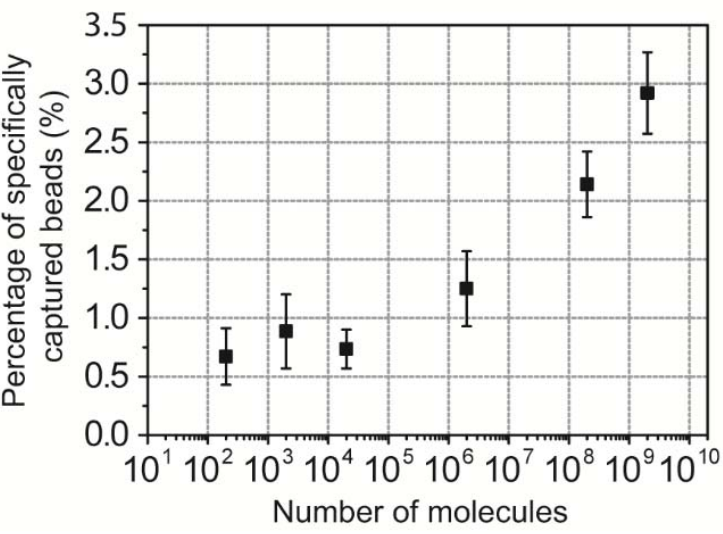

Figure 1. (a) Schematic representation of the time-lapse motion of a large bead exploring the detection area and interacting with the functionalized surface on the small beads through magnetic dipole-dipole forces. (b) Large bead capture mechanism: (bi) in absence of ligands, large beads are transported by the microfluidic flow through the whole detection area and washed away; (bii) if at least one ligand molecule is present on the large 10 bead surface, the bead is captured onto the detection area via ligand-receptor binding. (c) Assay readout: counting of the captured large beads provides a measure of the number of molecules present in the sample under analysis. (d) Experimental data for the detection of anti-streptavidin in Fetal Bovine Serum (FBS) (n=3-4, errors bars are $\mathrm{SD}$ ). 
a Flow

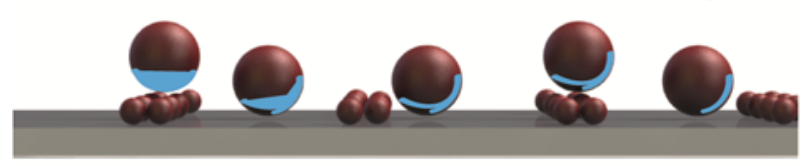

bi

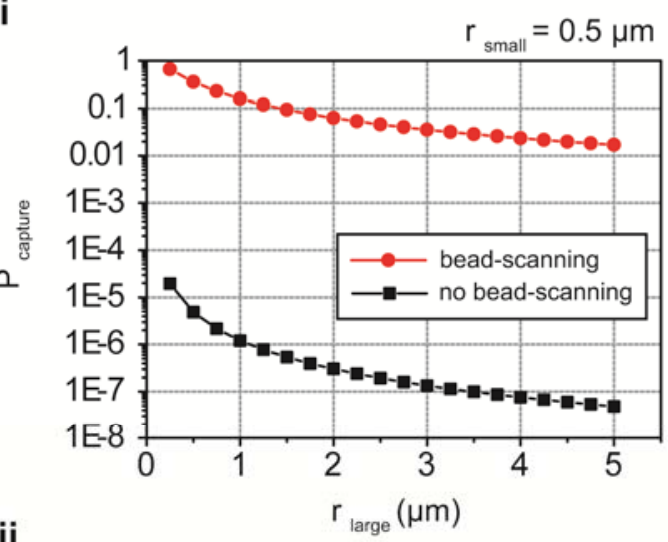

bii
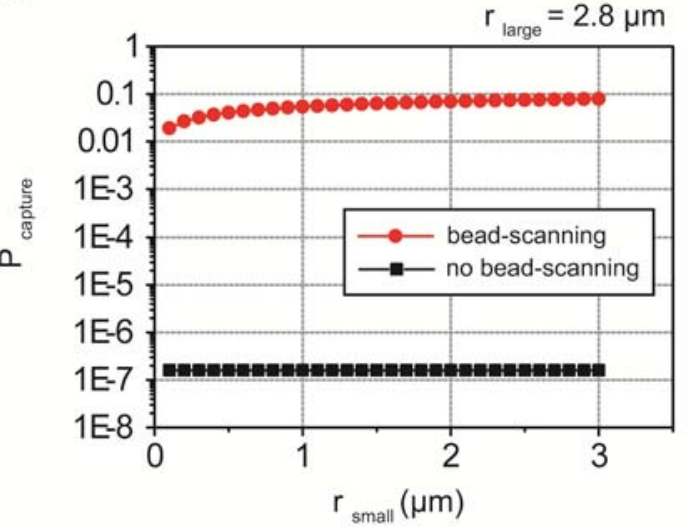

Figure 2. (a) Schematic representation of the bead-scanning mechanism: the orientation of the large bead changes at every encounter with a group of small beads and a progressively increasing portion of the large bead ${ }_{5}$ surface gets in contact with the functionalized surface of the small beads available for specific binding. (b) Theoretical estimation of the large bead capture probability $P_{\text {capture }}$ for 'bead scanning' and 'no bead scanning' conditions and for different sizes of both the (bi, $\left.r_{\text {small }}=0.5 \mu \mathrm{m}\right)$ large and the (bii, $\left.r_{\text {large }}=2.8 \mu \mathrm{m}\right)$ small beads. 
a

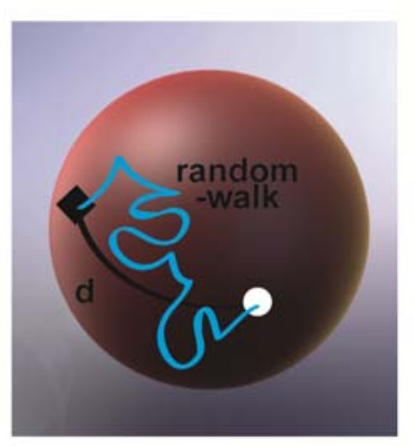

b

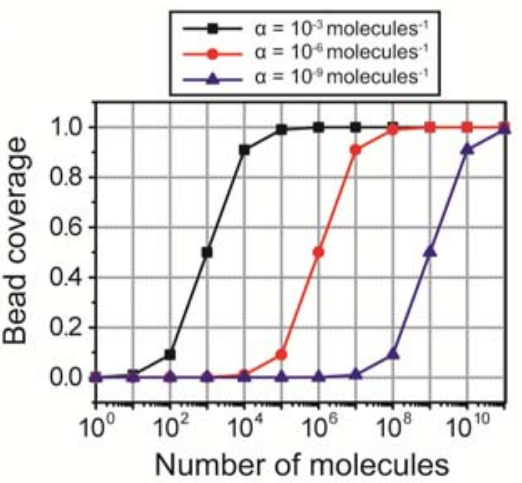

Figure 3. (a) Schematic representation of the random walk modeling approach: the contact point between large and small beads describes a random-walk on the surface of the large beads, from the initial contact point to the $s$ location of a captured ligand molecule. (b) Calculation of the large bead coverage of captured ligand molecules, assuming a Langmuir relationship for molecule capture, and different values of the parameter $\alpha\left(10^{-3}, 10^{-6}\right.$ and $10^{-9}$ molecule $\left.^{-1}\right)$. 
a

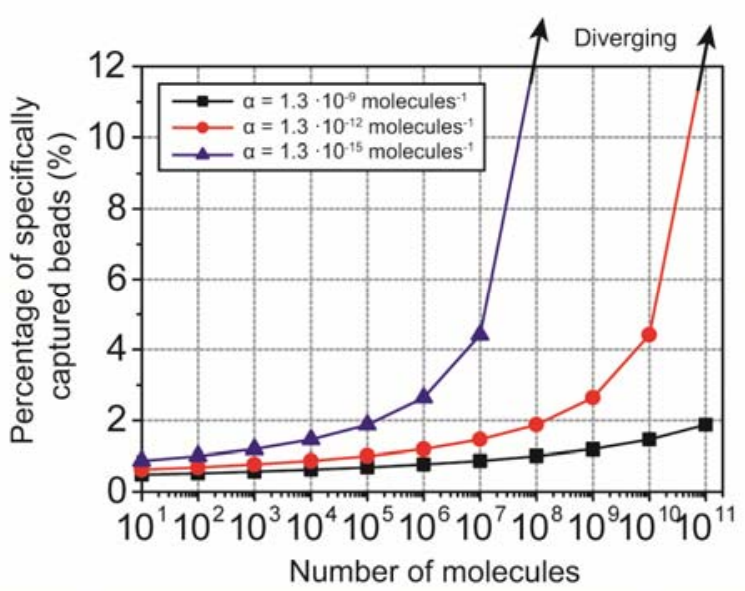

C

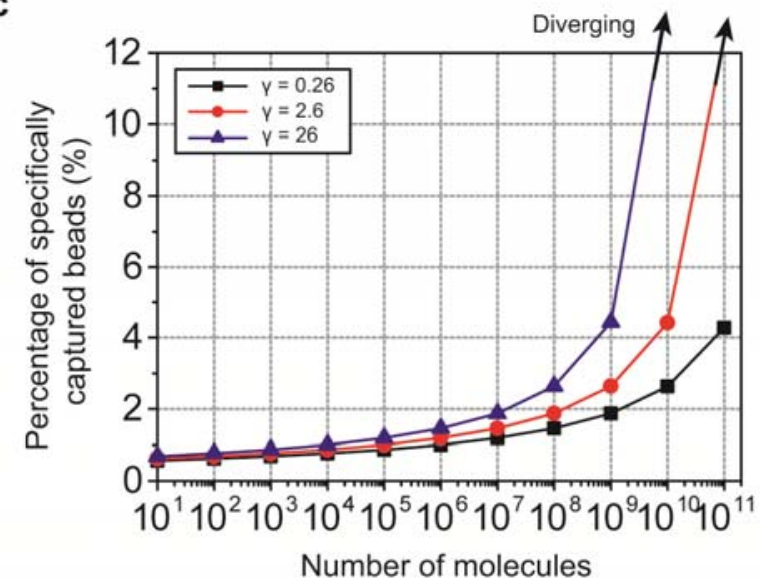

b

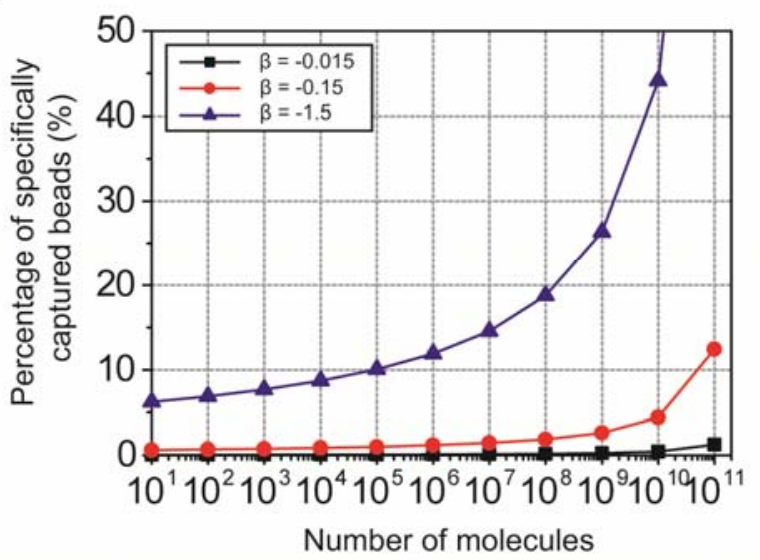

d

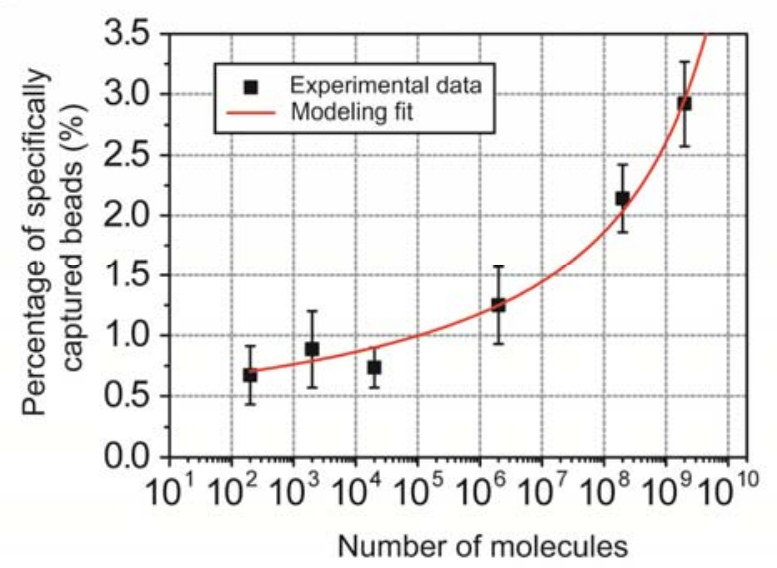

Figure 4. Plots of the function describing the assay readout (equation (16)), for different values of the fitting s parameters (a) $\alpha$ is a parameter, $\beta=-0.15, \gamma=2.6$, (b) $\beta$ is a parameter, $\alpha=1.3 \times 10^{-12}$ molecule $^{-1}, \gamma=2.6$, and (c) $\gamma$ is a parameter, $\beta=-0.15$ and $\alpha=1.3 \times 10^{-12}$ molecule $^{-1}$. (d) Comparison between the experimental data for the detection of anti-streptavidin in Fetal Bovine Serum (FBS), shown in Fig. 1(d), and the model predictions. Experimental data have been fitted using equation (16), yielding $\alpha=1.3 \times 10^{-12}$ molecule $^{-1}, \beta=-0.15$ and $\gamma=2.6$, with $\mathrm{R}^{2}=0.99$. 


\section{References}

1. H. C. Tekin, M. Cornaglia and M. A. M. Gijs, Lab Chip, 2013, 13, 1053-1059.

5 2. R. Etzioni, N. Urban, S. Ramsey, M. McIntosh, S. Schwartz, B. Reid, J. Radich, G. Anderson and L. Hartwell, Nature reviews. Cancer, 2003, 3, 243252.

3. C. D. Keating, Proc Natl Acad Sci U S A, 2005, 102, 2263-2264.

4. M. De, S. Rana, H. Akpinar, O. R. Miranda, R. R. Arvizo, U. H. F. Bunz and V. M. Rotello, Nat Chem, 2009, 1, 461-465.

5. N. L. Anderson and N. G. Anderson, Mol Cell Proteomics, 2002, 1, 845-867.

10 6. M. A. M. Gijs, Microfluid Nanofluid, 2004, 1, 22-40.

7. M. A. M. Gijs, F. Lacharme and U. Lehmann, Chem. Rev., 2010, 110, 1518-1563.

8. H. C. Tekin and M. A. M. Gijs, Lab Chip, 2013, 13, 4711-4739.

9. A. van Reenen, A. M. de Jong, J. M. J. den Toonder and M. W. J. Prins, Lab Chip, 2014, 14, 1966-1986.

10. S. P. Mulvaney, K. M. Myers, P. E. Sheehan and L. J. Whitman, Biosens. Bioelectron., 2009, 24, 1109-1115.

15 11. V. N. Morozov, S. Groves, M. J. Turell and C. Bailey, J. Am. Chem. Soc., 2007, 129, 12628-+.

12. S. J. Osterfeld, H. Yu, R. S. Gaster, S. Caramuta, L. Xu, S. J. Han, D. A. Hall, R. J. Wilson, S. H. Sun, R. L. White, R. W. Davis, N. Pourmand and S. X. Wang, P. Natl. Acad. Sci. USA, 2008, 105, 20637-20640.

13. R. S. Gaster, L. Xu, S. J. Han, R. J. Wilson, D. A. Hall, S. J. Osterfeld, H. Yu and S. X. Wang, Nat Nanotechnol, 2011, 6, 314-320.

14. R. S. Gaster, D. A. Hall, C. H. Nielsen, S. J. Osterfeld, H. Yu, K. E. Mach, R. J. Wilson, B. Murmann, J. C. Liao, S. S. Gambhir and S. X. Wang, Nat. 20 Med., 2009, 15, 1327-1332

15. H. C. Tekin, V. Sivagnanam, A. T. Ciftlik, A. Sayah, C. Vandevyver and M. A. M. Gijs, Microfluid. Nanofluid., 2011, 10, 749-759.

16. V. Sivagnanam, B. Song, C. Vandevyver and M. A. M. Gijs, Anal. Chem., 2009, 81, 6509-6515.

17. M. Cornaglia, H. C. Tekin, T. Lehnert and M. A. M. Gijs, J Appl Phys, 2013, 114.

18. M. Cornaglia, R. Trouillon, H. C. Tekin, T. Lehnert and M. A. Gijs, Anal Chem, 2014, 86, 8213-8223.

25 19. V. N. Morozov and T. Y. Morozova, Anal Chim Acta, 2006, 564, 40-52.

20. M. Cornaglia, R. Trouillon, H. C. Tekin, T. Lehnert and M. A. M. Gijs, Anal. Chem., 2014, 86, 8213-8223.

21. N. K. Lee, A. Johner, F. Thalmann, L. Cohen-Tannoudji, E. Bertrand, J. Baudry, J. Bibette and C. M. Marques, Langmuir, 2008, 24, $1296-1307$.

22. L. Cohen-Tannoudji, E. Bertrand, J. Baudry, C. Robic, C. Goubault, M. Pellissier, A. Johner, F. Thalmann, N. K. Lee, C. M. Marques and J. Bibette, Phys Rev Lett, 2008, 100.

30 23. S. Condamin, O. Benichou, V. Tejedor, R. Voituriez and J. Klafter, Nature, 2007, 450, 77-80.

24. E. B. Saff and A. B. J. Kuijlaars, Math Intell, 1997, 19, 5-11.

25. W. Habicht and B. L. van der Waerden, Math. Ann., 1951, 123, 223-234.

26. I. Langmuir, J Am Chem Soc, 1918, 40, 1361-1403.

27. I. Langmuir, J Am Chem Soc, 1932, 54, 2798-2832. 